\title{
C-QUAL: A system for computer-aided transcription of videotaped action sequences
}

\author{
STARKEY DUNCAN, JR., and RICHARD E. SAYRE \\ University of Chicago, Chicago, Illinois
}

C-QUAL is a system for the computer-aided transcription of action sequences from videotape. The system is designed for research on face-to-face interaction focusing on sequences of action involving speech and body motion. When the analyst indicates the beginning or the ending of a specified action, C-QUAL associates that event with the frame number on which it was transcribed and enters the information in a database. In this manner, all transcribed actions are located sequentially with respect to each other to the accuracy of a single video frame. Entered data can be superimposed on the video image for checking and editing. By using the SequelAnalysis Module, an innovative device for visually representing speech, C-QUAL permits speech transcription (including syllables, pauses, intonation, and paralanguage) to be fully integrated with body-motion transcription. The system supports the transcription of both speech and body motion in either tape direction or at any tape speed, including stop frame.

$\mathrm{C}-\mathrm{QUAL}$ is a response to data-generation problems encountered in research on the process of face-to-face interaction. The system is designed to deal with a major obstacle to ongoing work in this area-the considerable difficulty of generating accurate data on interaction sequences in the transcription of speech and body motion. It is hoped that facilitating the process of generating sequential data on face-to-face interaction will encourage both more widespread and more powerful analyses of this phenomenon.

Generating accurate sequential data is problematic for three reasons:

1. Manual transcription of action sequences is an arduous and time-consuming process at best. The investigator must observe and record not only the beginning and ending of each action but also its sequential location with respect to all other recorded actions.

2. Integration of speech with body-motion data is difficult because aural information must be combined with visual information. The problem is aggravated by the sensitivity of the sound track to tape speed and direction.

3. Entry of transcribed data into a computer database is relatively slow and is subject to clerical error. C-QUAL addresses these and other transcription problems.

C-QUAL may be relevant to the work of investigators in a number of areas related to language and interaction, such as the process of face-to-face interaction, the relation

Development of this system was supported in part by National Institute of Mental Health Grant MH-38344 and by grants from the Benton Education Research Fund, the NSF Biological Instrumentation Program, the ADAMHA Small Instrumentation Grant Program, and the IBM Corporation to Starkey Duncan, Jr. We extend our special thanks to David Breed for his invaluable assistance with the electronic aspects of the system. Requests for reprints should be sent to Starkey Duncan, Jr., Department of Psychology, University of Chicago, 5848 S. University Avenue, Chicago, IL 60637. of gesture to language production, language acquisition, animal behavior, the development of interactive competence, communication disabilities, and classroom interaction. More broadly, C-QUAL may be of interest to investigators concerned with generating sequentially accurate data from videotape, regardless of whether or not speech is involved. We shall describe the system in terms of its application to our own research-the process of face-toface interaction (Duncan \& Farley, 1990; Duncan \& Fiske, 1977; Duncan, Fiske, Denny, Kanki, \& Mokros, 1985), in which considerable emphasis is placed on the integrated analysis of speech and body motion.

\section{OVERVIEW}

Before C-QUAL is described in detail, a brief overview of the C-QUAL screen layout and the transcription process may be helpful. Figure 1 shows a C-QUAL screen during transcription. The videotape that is used would be a copy on which a representation of speech (Figure 1a) and machine-readable frame numbers have been inserted. In C-QUAL, transcribing can be done in either tape direction and at any available tape speed. For purposes of illustration, we shall say that the video image is displayed at stop frame. We shall also say that the analyst desires to transcribe to the accuracy of a single video frame.

The shaded area covering the greater part of the screen represents the video image. We invite the reader imaginatively to supply the color image. Computer-generated displays are superimposed on the video image. Across the top of the screen is a command line. At Figure $1 \mathrm{~b}$ is the transcribing menu entered by the analyst. In this case, the beginning and the ending of gazing at the partner are transcribed for Participants 1 and 2.

At the bottom of the screen are four lines. The lines typically scroll together from right to left in conjunction 


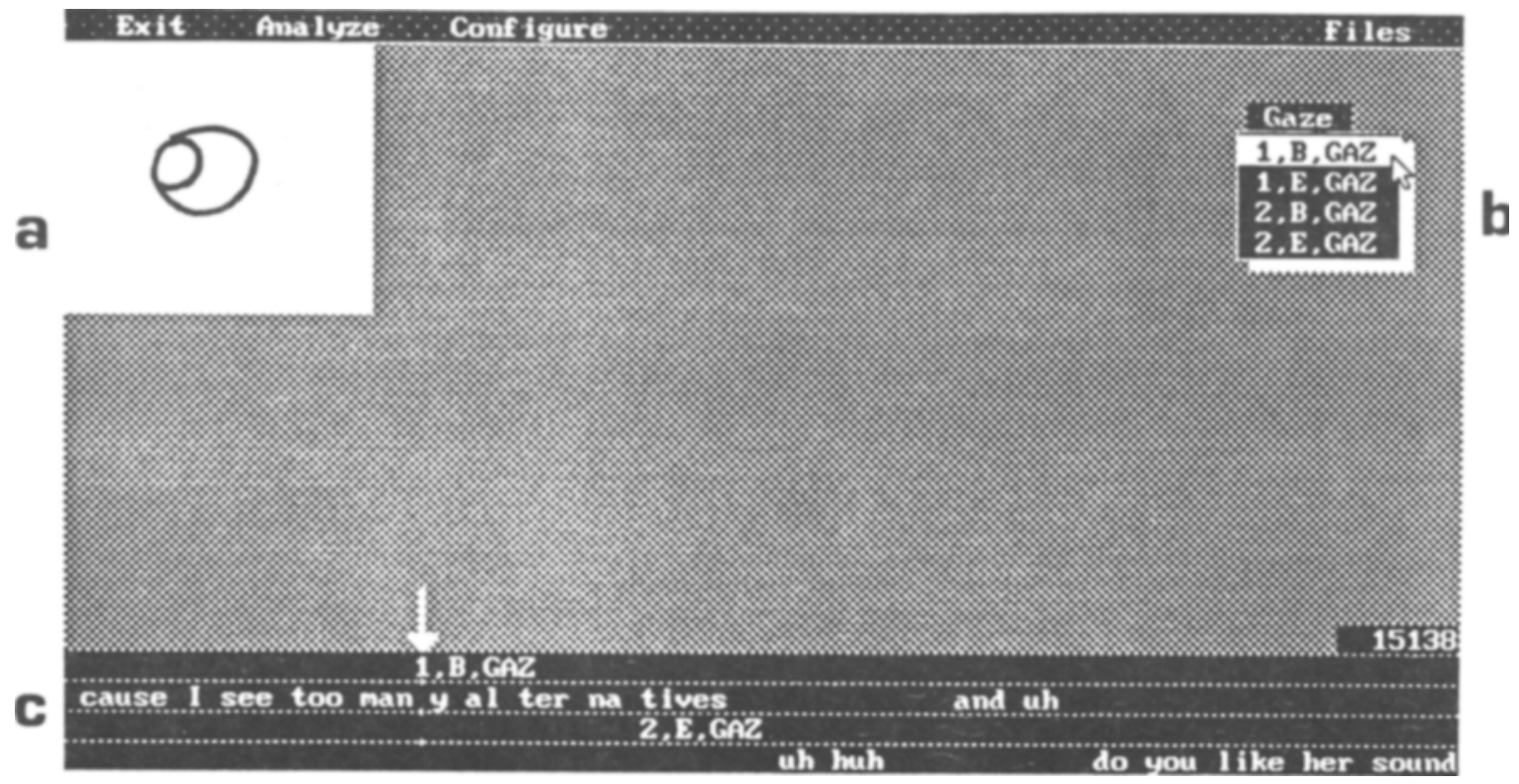

Figure 1. C-QUAL Transcribing Screen. (Video image is omitted. Sequel-Analysis Module display is simulated.)

with the videotape. In this case, they are arranged to provide the top two lines for Participant 1 and the bottom two for Participant 2. The top line for each participant shows the gazing that has been transcribed. The second line for each participant shows speech that has been eniered. The lines scroll under the vertical arrow, linking the current video frame to the transcription. The small box on the right, just above the four lines, shows the current frame number.

In transcribing, the analyst can view the tape in the direction and at the speed desired. When the beginning or the ending of an action (in this case, gaze) is detected, the analyst can stop and reposition the tape, stepping it forward or backward to identify the exact frame on which the action occurs. When that frame is reached, the analyst clicks on the menu item designating the action (in this case, 1,B,GAZ). The code appears under the arrow on the appropriate line at the bottom of the screen, as shown in Figure 1, and the code and frame number (15138) are entered in a computer database.

\section{DESIGN FEATURES}

In designing C-QUAL, the goal was for the computer to take over the most arduous and error-prone aspects of sequential data generation. Ideally, the investigator can transcribe by indicating via the computer only the onset and offset of actions observed on videotape.

1. Information on interaction sequences is generated by the computer. While viewing the tape, the analyst inputs the beginning and the ending of each action. The com- puter associates each such input with a machine-readable video frame number, thus identifying its temporal location on the tape. If desired, this information can be transcribed with maximum accuracy by stepping the tape forward frame by frame. In this manner, each transcribed action is located sequentially with respect to every other action to the maximum accuracy of a single video frame $(1 / 30 \mathrm{sec})$. This provides the sequential information essential for analyses of interaction.

2. Videotape analysis is possible at any speed, including stop frame, and in either tape direction. This consideration rules out the use of the SMPTE longitudinal time code that is recorded on the audio track.

3. Transcribing face-to-face interaction encompasses both speech and body motion. Although problems attending the transcribing of speech require special consideration, every effort is made to treat speech transcription in a manner as closely analogous to body-motion transcription as possible. Integrating speech and body-motion data and comparing these data in a transcription should be straightforward.

4. Perhaps the most unusual aspect of C-QUAL is the capability to analyze speech at any tape speed, including stop frame, and in both forward and reverse tape directions. This becomes possible with a special electronic device (SAM-259), which will be described later.

5. Transcribed information can be overlaid on the video image and displayed on the monitor so that the two can be directly compared.

6. Transcribed data are transparently collected in a computer data file. 
7. A full, printed transcription can be produced.

The process of transcribing with C-QUAL is not as simple as merely counting actions, but it is much closer to that than to the full-scale, manual transcription of interaction sequences.

\section{GALATEA}

C-QUAL is a direct descendant of GALATEA (Futrelle \& Potel, 1975; Potel, Sayre, \& MacKay, 1980), an earlier system developed at the University of Chicago. Implemented on a PDP-11/40 minicomputer and designed for biological research, GALATEA was an extraordinarily powerful tool for its time, anticipating many subsequent developments in image processing and interactive graphics.

Beyond the basic task of gathering data, GALATEA could generate animations of movements or changes of shape, including three-dimensional animations that could be viewed from any angle, and it could perform a number of calculations based on these data. Many of these functions are, of course, irrelevant to the problems of transcribing interaction that were mentioned earlier. In addition, GALATEA was unsuitable for human-interaction research because it could not handle speech information (it was originally designed for work on slime molds) and because it could be used only with movie film.

C-QUAL adapts concepts and techniques developed for GALATEA to current needs by its suitability for DOScompatible microcomputers, by the fact that it is designed for videotape recorders, and by its inclusion of speech transcription.

\section{HARDWARE}

\section{Time Code}

The foundation of any system of this type is the capability to write machine-readable frame numbers on the videotape. With these frame numbers, the computer can sense the current location on the tape, which spares the analyst the task of recording frame numbers or other time information and permits event-time accuracy to $1 / 30 \mathrm{sec}$.

Machine-readable frame numbers can be generated either by using longitudinal time code, which is written on the audio track, or by using SMPTE VITC (verticalinterval time code), which is written on the video portion of the tape. Longitudinal time code provides an adequate solution when the tape is viewed at normal or near-normal speeds. However, the use of longitudinal time code becomes more problematic when numbers are read while the tape is moving at very fast or very slow speeds, and, of course, it does not work at stop frame.

Thus, for more detailed analyses, VITC is superior, though more expensive. Currently, writing frame numbers on a tape requires making a copy of the tape and passing the original video signal through the frame-number generator, which adds frame numbers to the signal. For this reason, transcription via C-QUAL is always done on a second-generation tape. This arrangement requires that the investigator have access to equipment capable of producing high-quality videotape copies. Copying each tape in order to record time code is obviated if a different approach to machine-readable frame numbers is available, such as a laser disk, or if time code is recorded by the camera during taping. It seems reasonable to expect that this awkward process will be considerably simplified in the near future.

The current system uses an EECO VIG-850 intelligent time-code generator/reader.

\section{Other Hardware}

C-QUAL is implemented on a DOS-compatible microcomputer. C-QUAL is written in $\mathrm{C}$ and uses the Borland Turbo $\mathrm{C}$ compiler and the Metagraphics MetaWINDOW graphics library.

The overlay of computer-generated information (such as system status, menus, and transcribed actions) on the video image is accomplished by means of a Matrox VGO-AT-NI Enhanced Graphics/Text Overlay Board (noninterlaced) and a Mitsubishi Diamond Scan color monitor AUM-1381A.

We have been using a beta-format videocassette recorder because we regard its tape-handling features, including smooth changes in speed and direction, as superior. Video format is, of course, irrelevant to the system. Most other video formats can be used.

It is clear that the requirements of the system can be implemented with various combinations of compatible hardware.

\section{Sequel-Analysis Module}

The Sequel-Analysis Module (SAM-259; Frey, Hirsbrunner, Florin, Daw, \& Crawford, 1983), a unique ana$\log$ processor, greatly facilitates the transcription and recognition of speech when the tape is played in slow motion, stop frame, or reverse. This device was originally developed as a speech-perception aid for the deaf. SAM-259 receives the speech signal from the videocassette recorder and sends a transformed signal to an oscilloscope. The resulting display provides a representation of the speech, which permits various elements, particularly vowels, to be readily identified. Figure 2 illustrates the sorts of shapes generated by SAM-259. Because the device is sensitive to vowel quality, the shapes can vary with the speaker's language and, to some extent, with the dialect. The shapes, however, appear quite effective for identifying the vowels and certain consonants of a speaker.

With the use of a camera and video mixer, a video image of the oscilloscope display is inserted at an appropriate location on a copy of the videotape being analyzed (see Figure 1a).

Because a recognizable and undistorted speech representation is available when the tape is viewed, regardless of tape direction or speed, it becomes possible to treat speech in much the same manner as body motion. The investigator can mark the beginning and the ending of speech elements on the basis of visual information, just 
1

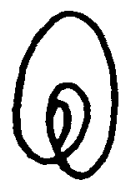

ฤิ

$\mathbf{s}$

a

$\mathbf{e}$

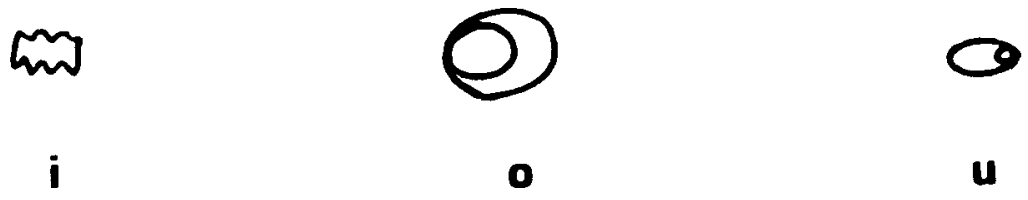

Figure 2. Examples of Sequel-Analysis Module (SAM-259) representations of speech sounds.

as the beginning and the ending of gestures or gaze are marked. This provides the investigator with unusual power and precision in treating speech and in linking speech to body motion in the transcription. Inquiries concerning SAM-259 can be made to the first author.

In recordings of naturalistic interaction, phenomena such as participants' talking at the same time and background noise may of course make this display more difficult to use. However, SAM-259 has a noise-suppression capability, and the speech signal can be further filtered before it is fed into the device.

\section{Notes on Equipment}

It will be apparent that the detailed analysis of videotaped interactions will require a relatively sophisticated videocassette recorder capable of slow motion, stop frame, and the like.

For investigators who are not pursuing integrated analysis of speech and body motion, the SAM-259 and its associated equipment will obviously not be necessary.

The frame-number generator writes its vertical-interval time code in the first lines of the video signal. It is necessary, therefore, to use a video mixer that passes the en-

Table 1

C-QUAL System Components

\begin{tabular}{|c|c|c|}
\hline Substitutable* & Functiont & System Component \\
\hline \multicolumn{3}{|r|}{ Software } \\
\hline No & $\mathrm{T}$ & C-QUAL, version 1 \\
\hline No & $\mathbf{T}$ & MS-DOS, Version 3.30 or higher \\
\hline No & $\mathbf{T}$ & MetaWINDOW, Version 3.4b \\
\hline \multicolumn{3}{|r|}{ Hardware } \\
\hline Yes & $\mathrm{T}$ & IBM PC equivalent with AT bus; $640 \mathrm{~K}$ RAM and hard disk \\
\hline Yes & $\mathrm{T}$ & Monochrome display adapter and monitor \\
\hline No & $\mathbf{T}$ & Matrox VGO-AT/NI display adapter with video overlay capability and monitor \\
\hline Yes & $T$ & mouse \\
\hline Yes & $\mathrm{T}$ & printer \\
\hline Yes & $T$ & serial port (for time code) \\
\hline Yes & $\mathrm{T}$ & RS422-RS232 converter \\
\hline No & $\mathrm{T}, \mathrm{C}$ & EECO VIG-850 video time-code writer/reader \\
\hline Yes & T, C & Prime Image TBC $\cdot$ sync + video timebase corrector \\
\hline Yes & $\mathrm{T}, \mathrm{C}$ & Sony SL-2S00 Betamax VCR and remote control \\
\hline Yes & C & second VCR for recording time code and SAM-259 \\
\hline Yes & $\mathbf{S}$ & Panasonic WJ-4600C video mixer \\
\hline No & $\mathbf{S}$ & Sequel-Analysis Module (SAM-259) \\
\hline Yes & $\mathbf{S}$ & Hitachi model V-523, $50 \mathrm{MHz}$ oscilloscope \\
\hline Yes & $\mathbf{S}$ & audio equalizer \\
\hline
\end{tabular}

*Components for which equivalents are believed to be easily substituted. $+\mathrm{T}=$ transcribing; $\mathrm{C}=$ timecode processing; $\mathbf{S}=$ speech visualizing. 
tire video signal. Otherwise, the frame-number information will not be included in the mixer's output signal.

Table 1 lists hardware and software components of C-QUAL, as currently implemented. Components for which, to the best of our knowledge, equivalent alternatives may be substituted are indicated.

\section{TRANSCRIBING SPEECH}

C-QUAL makes three simultaneous representations of speech available to the investigator: audio track, SAM-259 image, and analyst-entered speech transcription. They can be used singly or in combination to generate accurate, high-resolution transcriptions at various levels of detail.

For example, the boundaries of major speech units may be marked, omitting detailed phonological information. Thus, one might indicate boundaries of phonemic or syntactic clauses, sentences, speech acts, or speaking turns, together with information on the type of unit, such as Participant 1's speaking turn or a request speech act. Playing the tape at normal or near-normal speeds, the investigator can locate a major boundary, such as the exchange of a speaking turn. The tape can then be positioned just before that boundary and stepped forward. By observing the inserted SAM-259 image, the investigator can identify the frames on which one speaking turn ends and the next begins. These events can be transcribed by using the data-entry menu. C-QUAL combines this information with the body-motion transcription, so that, for example, one can study the relation of gaze direction or gestures to speech-act requests or turn exchanges.

A more detailed transcription of speech can be achieved within C-QUAL by using a procedure such as the following:

1. On a word processor, make a transcription of the desired speech elements for each participant, perhaps using an audio tape recorder.

2. The word-processor file can be imported by C-QUAL. As illustrated in Figure 1, speech for one participant will be on one line at the bottom of the screen, and speech for the partner will be on another line. (The other two lines are for displaying information on a second set of actions. In this case, one might choose intonation-e.g., stress, pitch, and contour-as illustrated in Figure 3a.)

3. Construct a data-entry menu specifying that the beginning and the ending of the desired speech elements will be transcribed.

4. The transcription is then scrolled in either direction under the control of the analyst. The vertical arrow indicates the current video frame. (The arrow is positioned in the display so that the investigator can read elements of the transcription preceding and following the current speech element.) Scroll the transcription to the beginning of the first element to be transcribed.

5. By means of the sound track and the SAM-259 image, identify the frame on which the element begins and transcribe this information by using the data-entry menu. The occurrence of the indicated action is entered in the database together with its accompanying frame number.
6. Once this process is begun, the analyst can step the video tape forward frame by frame and scroll the transcription forward character by character. By using the SAM-259 image, each successive element can be identified and its beginning and ending entered in C-QUAL.

This transcription process provides C-QUAL with information on the location of each element, the number of frames that each element occupied, and the length of pauses between elements. A speech transcription is entered in C-QUAL that is accurate to $1 / 30 \mathrm{sec}$, that is not dependent on the analyst's response time in stopping and starting the videotape, and that can be tied to other events on the videotape such as gestures and gaze.

In the process of displaying the transcription, C-QUAL calculates the durations of the speech pauses. This information can be automatically inserted in the transcription and become part of the scrolling display. In the current version of C-QUAL, pause-duration information is represented as follows: . for $1 / 5 \mathrm{sec},+$ for $1 \mathrm{sec}$, and $-\mathrm{x}-$ for a longer pause of $x \mathrm{sec}$. An example of this approach would be: "uh huh....uh-15-what do you +++ think." Principles of treating pause information are further considered in the final section.

Many studies, of course, will not need information on the duration of each speech element and pause. This example illustrates, however, the manner in which elements of speech, chosen by the investigator, can be transcribed in C-QUAL.

Once the speech elements are entered, further information on intonation and paralanguage can be added as needed.

An example of speech transcription is shown in Figure 1c. In this example, one line displays the transcription of speech syllables, and one line displays the transcription of another specified action, gaze. Transcribed intonational stress, pitch, and contours can be specified, as indicated in Figure 3a.

When speech or other actions are entered in C-QUAL and linked to the frame numbers, the transcription will scroll across the bottom of the screen in sync with the video image, permitting cross-checking and verification. The scrolling is such that the current transcribed element is positioned below the arrow.

The orthographic representation of speech in Figures 1 and 3 is not a requirement of the system; speech representation is specified by the investigator. For example, phonetic, phonemic, or foreign-language representations could be used.

\section{Data Analysis}

C-QUAL is designed to produce computer data files that are ready for analysis. Investigators will have programs appropriate for their particular studies of action sequences. To investigators concerned with detecting recurrent sequential patterns in the data, we would mention THEME (Magnusson, 1989), a sophisticated program designed for that purpose. C-QUAL is capable of producing data files directly analyzable by THEME. For further information on THEME, investigators should contact the first author. 

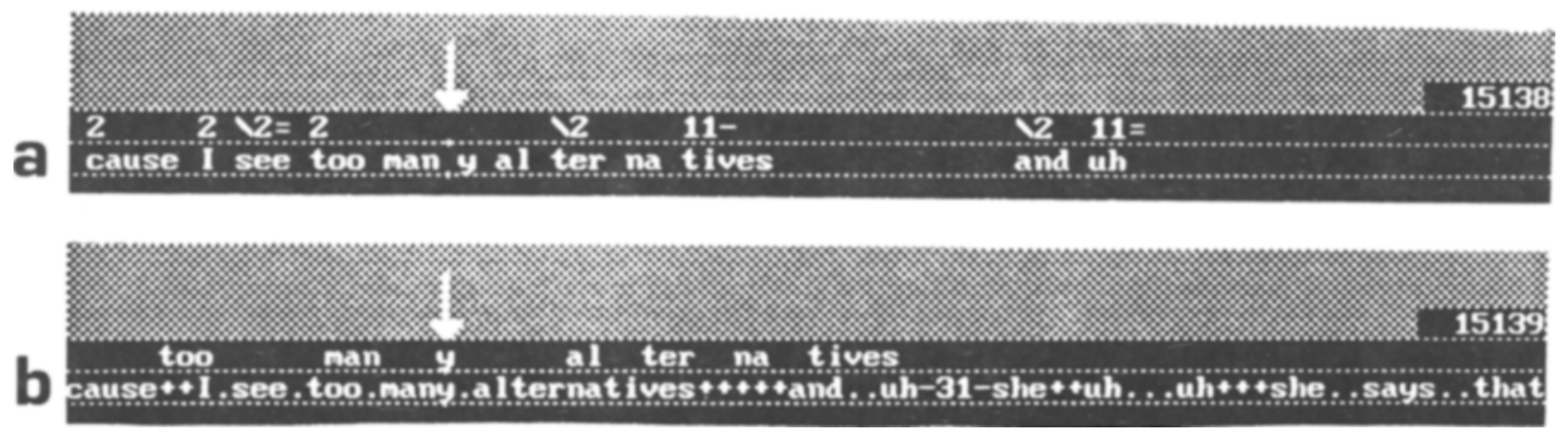

Figure 3. Scrolling transcription lines at bottom of C-QUAL overlay. (a) Speech syllables and intonation. (b) Linear (upper line) and nonlinear (lower line) representations of speech.

\section{REPRESENTATIONS OF THE DATA}

The computer's ability to translate rapidly between alternate representations of the same underlying data set permits the use of different representations that are chosen to suit particular tasks. Depending on the current task, a C-QUAL data set is represented in various forms that were specifically designed to aid the analysis process: (1) internal system representation, (2) ASCII file, (3) current transcription display synchronous with the video, (4) previous transcription display synchronous with the video, (5) simulated time mode, and (6) printed transcript. These will be considered in turn.

Internal representation. Regardless of the representation that is used at any given point in the transcribing process, the underlying form for each datum in C-QUAL is a pair $(f, c)$, the members of which represent discrete events: $f$ is a frame number representing the time of the event and $c$ is a code or character string describing the event. To permit rapid display and flexible access, as well as editing, the internal data format is a doubly linked list maintained in order by frame number. The internal data format is not directly accessible to the user but is basic to the interactive graphics capabilities provided by the system.

ASCII file. One of the alternate forms in which data may be represented is a simply structured file of lines of ASCII characters, as illustrated in Table 2 . The codes in Table 2 conform to the THEME format: 1 and 2 refer to participants, $B$ and $E$ refer to beginning and ending, and GAZ is the code for a participant's gazing at the partner. Code elements are separated by commas. This form is used in storing data on disk and in transferring these data to other computer systems, such as a spreadsheet, a statistical program, or THEME, for further processing and analysis.

Transcription display. During data capture, it is important that portions of the previously entered data are displayed to guide the investigator's activities. This display is usually synchronized with the video image, but it can be screened independently of the video. The ability to view and review previously entered data in synchrony with the videotape makes detection and correction of errors and omissions an inherent part of data entry rather than a laborious extra step. Thus, this design feature acts as an editor for transcripts.

Once an event has been entered, it can be displayed in a horizontal scroll at the bottom of the screen, either overlaid on the video image or on an adjoining monitor. In this discussion, we shall assume that it is overlaid (see Figure 1c). An arrow represents the current frame number. Transcribed events are scrolled horizontally in synchrony with the video, so that events transcribed for the current frame number are positioned below the arrow. Then, for example, deleting the event from the transcript could be accomplished by repositioning the video to $f$ and selecting, by mouse or keyboard, the menu item " $1, B, G A Z$."

Simulated time mode. Simulated time mode provides an alternate method of controlling the representation of time in the system. In this mode, the current time is not directly coupled to the video, permitting the investigator to review the data already entered without moving the VCR. Keyboard or mouse actions provide control of simulated frame rate, direction, position, and the like. This circumvents limitations of the VCR such as slow "fast forward" or awkwardness in finding a particular frame.

Table 2

Simply Structured ASCI File Resulting from C-QUAL Transcription

\begin{tabular}{cl}
\hline Frame No. & Event \\
\hline 1476248 & "BEGIN SECOND TAPE OF SULLIVAN FAMILY (5/4/90) \\
1582224 & $1, B, G A Z$ \\
1592240 & $2, B, G A Z$ \\
1594440 & 2, E,GAZ \\
1595006 & 1, E,GAZ \\
\hline
\end{tabular}


Printed transcript. C-QUAL can produce a printed transcript that displays transcribed data. In some cases, such a transcript could be considered as the final result, as would be the case in a preliminary study in which data analysis begins with the inspection of the transcript.

\section{ISSUES OF SYSTEM DESIGN}

Although its development was motivated by problems of transcribing action sequences in face-to-face interaction, C-QUAL is designed more generally to facilitate the generation of many kinds of sequential information from videotape. It is well adapted to record a number of different actions during separate passes of the videotape, where those passes can be at any speed and in either direction.

The program itself is intended to be as general as possible so that other investigators might adapt it to their needs. We hope the program will be shared and developed within the broader research community. As C-QUAL and similar programs are developed, research on faceto-face interaction and other sequential processes may be facilitated.

Hardware is obviously a basic consideration in developing a system of this sort. Unfortunately, a major obstacle has been finding appropriate hardware in a price range accessible to us and presumably to other investigators. We do not regard several aspects of the present hardware configuration as optimal. It seems reasonable to expect that hardware will continue to improve and that prices will continue to decline. C-QUAL is designed to be receptive to new hardware that improves on that currently available.

Most of the program is implemented in C, which facilitates transportability between computers. Although the graphics-display portions of a program are difficult to transport to alternative hardware, we hope this problem is mitigated by a packaged set of display programs. Displays in C-QUAL are generated through the MetaWINDOW graphics package, a product of Metagraphics Software Co. This package currently supports more than 100 different forms of display hardware and will probably be extended by its vendor to support new hardware as it becomes available.

\section{An Editor for Transcripts}

Considering the purpose of C-QUAL, it would seem logical to develop it by designing first a data-entry system and then an editor for corrections and revisions resulting from the inevitable mistakes and changes of opinion by the analyst. We have taken the opposite approach, designing an editor that incorporates data entry. Rather than separating data entry and editing, the required capabilities are integrated in a single program, the editor.

A good editor is an effective data-entry tool, but it extends beyond simple data entry to include, for example, the capacity to refer to a previously entered item in order to delete it. Therefore, an editor needs to be adapted both to the structure of the transcript and to the basic task of transcribing from videotape. Transcribing, checking, and amending need to be as easy and natural as possible.

Providing the display of the transcript in close proximity to the video-that is, either on an adjacent monitor or overlaid on the video image-permits the investigator to make a direct comparison of the transcription with the original. When the transcription is produced on the monitor in synchrony with the video image, a reference to the current time in the transcript is equivalent to a reference to the corresponding time in the video or audio. That is, the transcription and video index each other. This dual reference, or dual deixis, is exploited to make it easy for the analyst to refer to particular times in the transcript or in the video and to examine temporal relationships between the two.

In systems designed for entering spatial information, similar techniques have been used (Potel, Sayre, \& MacKay, 1980). The "transcript" is overlaid on the original image while an analyst traces the $(x, y)$ positions of objects of interest. That technique has been described as data entry by tracing. The current version of C-QUAL has no capacity for spatial information (of potential interest in the study of gestures, proxemics, and the like). C-QUAL registers the transcribed event codes with the video only in the dimension of time. Hence, the analogy to tracing is not directly applicable to C-QUAL. The more abstract category of dual deixis describes the essential strategy, designed to simplify the analyst's task, that these systems share.

Among the key characteristics of any editor are the methods it provides for referring to the objects edited. Usually, this is reduced to the problem of referring to a position within the text. For example, in line-oriented text editors, the user refers to line numbers or character positions to position a cursor at a character to be deleted. This is indirect and cumbersome, requiring the user to pay attention to an artificial scheme of line numbers rather than to the text itself.

Screen-oriented text editors are usually preferred, permitting cursor positioning via keyboard or mouse without reference to line numbers. The user still positions a cursor but views only the more concrete objects of the cursor, the text, and their relative positions.

A third option is to refer to a textual object by searching for a string, for example, "Find: 123," which would position the cursor at the end of the first occurrence of the string " 123 ." We provide these examples merely to clarify what we mean by capabilities for reference within an editor.

Considered as an editor, C-QUAL provides two alternate and rather unusual forms of reference to positions in the transcript. One form is reference by positioning in the time domain, either by control of the VCR or via the keyboard in simulated time mode. The other form is reference by dual deixis, wherein the analyst refers to the transcript by referring to the video or vice versa. The incor- 
poration of these two capabilities makes C-QUAL an editor that is particularly well suited to the task of transcription and subsequent checking from the video.

\section{Linear and Nonlinear Speech Representation}

The question of how to represent speech transcription on the screen raises interesting issues for a system of this sort. Simple linear relationships between time and display space, such as one character for $1 / 30 \mathrm{sec}$, can seldom be used. On a display showing 80 characters per line, only $80 / 30=2.67 \mathrm{sec}$ of speech data can be displayed. Further, if 5 characters per event are allocated (e.g., 5 characters to represent a syllable), only $80 / 5 / 30=.53 \mathrm{sec}$ of data can be displayed. If the time intervals are small, such as video frames at $1 / 30 \mathrm{sec}$, displays using a linear relationship of time to space are mostly blank. If the time intervals are large, then several events, for example, several syllables, may occur within a single interval, inconveniently requiring more characters per interval.

Our approach was to adopt a nonlinear representation of speech, which we described in the discussion of speech transcription. Pauses are represented explicitly as characters and are compressed. As we indicated earlier, our current coding scheme uses . for a pause of $1 / 5 \mathrm{sec},+$ for $1 \mathrm{sec},-15-$ for a 15-sec pause, and so on. As transcription proceeds, C-QUAL calculates these pauses and inserts the appropriate symbols in the speech display. We believe this procedure produces a readable and practical display.

Figure $3 b$ illustrates linear and nonlinear representations of speech. The upper line shows a linear representation, with spaces indicating absolute time between speech events. The lower line shows a nonlinear representation, with symbols indicating time between speech events.

We continue to experiment with such alternatives. C-QUAL, long in development, continues as an evolving system.

\section{REFERENCES}

Duncan, S. D., JR., Farley, A. M. (1990). Achieving parent-child coordination through convention: Fixed-and variable-sequence conventions. Child Development, 61, 742-753.

Duncan, S. D., JR., Fiske, D. W. (1977). Face-to-face interaction: Research, methods, and theory. Hillsdale, NJ: Erlbaum.

Duncan, S. D., JR., Fiske, D. W., Denny, R., Kanki, B. G., \& Mokros, H. B. (1985). Interaction structure and strategy. New York: Cambridge University Press.

Frey, S., Hirsbrunner, H.-P., Florin, A., Daw, W., \& Crawford, R. (1983). A unified approach to the investigation of nonverbal and verbal behavior in communication research. In W. Doise \& S. Moscovici (Eds.), Current issues in European social psychology (pp. 143-199). Cambridge, U.K.: Cambridge University Press.

Futrelle, R. P., \& Potel, M. J. (1975). The system design for GALATEA, an interactive real-time graphics system for movie and video analysis. Computers \& Graphics, 1, 115-121.

Magnusson, M. S. (1989). Structures syntaxiques et rythmes com: portementaux: Sur la détection de rythmes cachés. Science et Techniques de l'Animal du Laboratoire, 14, 143-147.

Potel, M. J., SAyre, R. E., \& MacKay, S. A. (1980). Graphics input tools for interactive motion analysis. Computer Graphics \& Image Processing, 14, 1-23.

(Manuscript received July 11, 1990; revision accepted for publication May 9, 1991.) 